ISSN 1936-5098

CAE Working Paper \#07-09

\title{
An Overlapping-Generations Model with Search
}

by

Tao Zhu

May 2007 


\title{
An overlapping-generations model with search*
}

\author{
Tao Zhu
}

May 16, 2007

\begin{abstract}
Search is embedded in an overlapping-generations model. The young participate in a centralized market, and then are matched in pairs in a decentralized market. The old only participate in the centralized market. If the buyer's bargaining power is sufficiently close to unity in pairwise trade and if the old are risk averse, the golden-rule rate of money transfer is positive. Such risk aversion, the pairwise trade, and dependence of the young's saving on the rate of return are necessary for this result.

JEL classification: E30, E31, E40, E41

Keywords: overlapping-generations; search; money creation; golden rule
\end{abstract}

\section{Introduction}

I develop a model of money with two frictions that usually do not appear in the same model: the overlapping-generations (OLG) friction and the search friction. In this model, people live for two periods. In each period, the young and the old participate in a centralized market; then the old die and the young are matched in pairs in a decentralized market. ${ }^{1}$ This setup is tractable,

\footnotetext{
*I am indebted to Neil Wallace for many useful comments and suggestions. I also thank Karl Shell for helpful conversations.

${ }^{1}$ Maeda [9] and Russell [12] study different OLG models with search. In neither is there centralized trade.
} 
because, as in any two-period-lived OLG model, the old sell all their money which implies that the inherited distribution of wealth induced by pairwise trade is not a state variable. ${ }^{2}$ Moreover, the model can be specified so that the pairwise meetings are necessary for money to be valued.

There was a debate about whether the ordinary OLG model is a suitable model of money. Some argued that the model is misleading because of the absence of a transactions role of money (see, e.g., Tobin [14]). One response was to add cash-in-advance or money-in-the-utility-function (see, e.g., McCallum [10]). Here, a transactions role of money is incorporated using pairwise meetings and anonymity.

This model has new implications for the optimal rate of lump-sum money creation compared to the ordinary OLG model and the OLG model with money-in-the-utility-function (see, e.g., Abel [1]); optimality here means maximization of the steady-state expected lifetime utility-golden-rule optimality. In particular, if the buyer's bargaining power in pairwise trade is close to unity and if the old are risk averse, then the golden-rule rate of money creation is positive. Such risk aversion, the pairwise trade, and dependence of the young's saving on the rate of return are necessary for this result.

To see why a positive transfer can be optimal, let buyers have all the bargaining power and consider a small reduction in the zero-inflation steady state saving (in the form of money) of one young person. The first-order effect from this reduction on his own lifetime expected utility is zero. Now consider his partner in a pairwise meeting. If the partner is the seller in the meeting, then she receives the same utility from trading with this person and all other buyers. But if the trading partner is the buyer in the meeting, then she receives more utility from trading with this person than from other sellers for the following reason. Because the old are risk averse, the postpairwise-meeting value function of money is strictly concave. And because this seller is poorer than other sellers, this strict concavity implies that he produces more than other sellers for the same payment. This leads to a better payoff for the trading partner. Therefore, there is a first-order effect from the person's reduced saving to the lifetime utility of others, an externality. Money creation that comes about through lump-sum transfers to the young produces such reduced saving and, therefore, is beneficial. As the above explanation suggests, both risk aversion for the old and the pairwise meetings

\footnotetext{
${ }^{2}$ This feature is exploited by Zhu and Wallace [16, section 3] to complete a generalequilibrium analysis. The version there is special in that the young's saving is exogenous.
} 
are necessary for this effect to occur.

The rest of the paper is organized as follows. In section 2, I describe the model and equilibrium and show existence. I establish results about the golden-rule rate of money creation in section 3 . In section 4 , I discuss introducing capital into the model, and some insight from this model that may carry over to the model of Lagos and Wright [8].

\section{The model}

In this section, I describe the model, define equilibrium, and show existence.

\subsection{Environment}

Time is discrete, dated as $t \geq 0$. Each date has two stages, 1 and 2 . At the start of each $t$, there is a unit mass of newly-born people, and each person lives for three consecutive stages. The person is young at date $t$ or his first two stages, and old at date $t+1$ or his third stage. There is one produced and perishable good per stage. There is another durable and intrinsically useless object called money; the initial old - those who die at the end of date 0 stage 1 -hold the initial money stock $M_{0}$.

A person born at $t \geq 0$ can produce but cannot consume at his first stage, and he can consume but cannot produce at his third stage. At his second stage, he has an equal chance to be a buyer - who can consume but cannot produce, or a seller - who can produce but cannot consume. At his $i$ th stage, his utility from consuming $q_{i}$ (given he can) is $u_{i}\left(q_{i}\right)$, and his disutility from producing is $q_{i}$ (given he can) is $c_{i}\left(q_{i}\right)$. I assume throughout, unless specified otherwise, that $c_{i}^{\prime}>0, c_{i}^{\prime \prime} \geq 0, u_{i}^{\prime}>0$, and $u_{i}^{\prime \prime} \leq 0$. The person's lifetime utility is the sum of his stage utility, and he maximizes his expected lifetime utility. $^{3}$

At stage 1 of date $t$, the young and old meet in a centralized spot market, and each young receives $\tau M_{t}$ with $\tau>-1$ units of money from the government, where $M_{t}$ is the average holding of the old at the start of $t$. At stage 2

\footnotetext{
${ }^{3}$ In this setting, if the person's second stage is removed and $c_{1}(q)=U(\omega)-U(\omega-q)$ and $u_{3}(q)=V(e+q)$, then the model is equivalent to the ordinary two-period-lived OLG model in which each person is endowed with $\omega$ when young and $e$ when old, and has the utility function $U$ when young and $V$ when old. Some variants of this setting are discussed in section 4 .
} 
of date $t$, the young are matched in pairs; matching is random but, without loss of generality, a buyer always meets a seller.

People are anonymous so money has an essential role in facilitating trade. The trade in the centralized market is competitive. In each pairwise meeting, each person's money holding is common knowledge, and the surplus from trade is split by generalized Nash bargaining.

\subsection{Definition of equilibrium}

Let $\rho_{t}$ be the real balance held by the old (hereafter, the real balance when the context is clear) in the date $t$ centralized market; that is, $\rho_{t}$ is the product of $M_{t}$ and the market price of money in term of goods. Let $\theta \in(0,1]$ be the buyer's bargaining power in pairwise meetings. Also, let the individual state $x$ be the ratio of the individual money holding to the current stock of money.

Next I describe the individual choice problems by backward induction, starting with the end of date $t$. A person who enters old age in state $x$ will receive the payoff $u_{3}\left(x \rho_{t+1}\right)$. Now, consider a date $t$ pairwise meeting between a buyer in state $z$ and a seller in state $y$. A trade $(q, l)$, where $q$ is the transfer of the good and $l M_{t+1}$ is the transfer of money, will give the buyer a surplus $u_{2}(q)+u_{3}\left(z \rho_{t+1}-l \rho_{t+1}\right)-u_{3}\left(z \rho_{t+1}\right)$, and the seller a surplus $-c_{2}(q)+u_{3}\left(y \rho_{t+1}+l \rho_{t+1}\right)-u_{3}\left(y \rho_{t+1}\right)$. Therefore, the trade reached in the meeting, denoted $\left(q\left(z, y ; \rho_{t+1}\right), l\left(z, y ; \rho_{t+1}\right)\right)$, is a maximizer of the problem

$$
\begin{aligned}
& \max _{q \geq 0,0 \leq l \leq x}\left[u_{2}(q)+u_{3}\left(z \rho_{t+1}-l \rho_{t+1}\right)-u_{3}\left(z \rho_{t+1}\right)\right]^{\theta} \\
& \times\left[-c_{2}(q)+u_{3}\left(y \rho_{t+1}+l \rho_{t+1}\right)-u_{3}\left(y \rho_{t+1}\right)\right]^{1-\theta}
\end{aligned}
$$

The buyer's payoff is

$$
f\left(z, y ; \rho_{t+1}\right)=u_{2}\left(q\left(z, y ; \rho_{t+1}\right)\right)+u_{3}\left(z \rho_{t+1}-l\left(z, y ; \rho_{t+1}\right) \rho_{t+1}\right),
$$

and the seller's payoff is

$$
g\left(z, y ; \rho_{t+1}\right)=-c_{2}\left(q\left(z, y ; \rho_{t+1}\right)\right)+u_{3}\left(y \rho_{t+1}+l\left(z, y ; \rho_{t+1}\right) \rho_{t+1}\right)
$$

Hence, before stage-2 pairwise meetings, the expected utility of a young person in state $x$ is

$$
v\left(x ; \rho_{t+1}, \mu_{t}\right)=0.5 \int\left[f\left(x, y ; \rho_{t+1}\right)+g\left(y, x ; \rho_{t+1}\right)\right] \mu_{t}(d y),
$$


where $\mu_{t}$ is the distribution of young individual states just before stage-2 pairwise meetings. ${ }^{4}$ Hence, the young person's problem in the date $t$ centralized market is

$$
\max _{l \geq \max \{0,-\tau\}}-c_{1}\left(l \rho_{t}\right)+v\left(\frac{l+\tau}{1+\tau} ; \rho_{t+1}, \mu_{t}\right) .
$$

Now let $X\left(\rho_{t}, \rho_{t+1}, \mu_{t}\right)$ be the set of the (young) individual states consistent with individual optimal choices; that is, let

$$
X\left(\rho_{t}, \rho_{t+1}, \mu_{t}\right)=\left\{\frac{l+\tau}{1+\tau}: \text { some }(q, x) \text { solves }(5)\right\} .
$$

The support of $\mu_{t}$, denoted supp $\mu_{t}$, must be a subset of $X$, and market clearing requires $\int x \mu_{t}(d x)=1$ (notice that $X$ need not be a singleton because $v$ need not be concave). Then, we have

Definition 1 A sequence $\left\{\left(\rho_{t}, \mu_{t}\right)\right\}_{t=0}^{\infty}$ is a monetary equilibrium if $\rho_{t}>0$, $\int x \mu_{t}(d x)=1$, and supp $\mu_{t} \subset X\left(\rho_{t}, \rho_{t+1}, \mu_{t}\right), \forall t \geq 0$. A pair $(\rho, \mu)$ is a monetary steady state if the sequence $\left\{\left(\rho_{t}, \mu_{t}\right)\right\}_{t=0}^{\infty}$ with $\left(\rho_{t}, \mu_{t}\right)=(\rho, \mu) \forall t$ is a monetary equilibrium.

\subsection{Existence of a steady state}

Existence can be established under fairly general assumptions.

Proposition 1 Suppose $c_{1}^{\prime \prime}>0, c_{1}^{\prime}(\infty)=\infty, c_{2}(q)=q, u_{2}(0)=0, u_{2}^{\prime}(0)=$ $\infty$, and $\left[u_{2}(x) / u_{2}^{\prime}(x)\right]\left[u_{2}^{\prime \prime}(x) / u_{2}^{\prime}(x)\right](1-1 / \theta) \equiv h(x)$ is bounded in a neighborhood $x=0$. Then there exists a Definition-1 steady state.

Proof. See the appendix.

The assumptions in Proposition 1 are maintained throughout unless explicitly noted. The boundedness condition on $h$ holds if $\theta=1$ and is otherwise satisfied if $u_{2}$ is a power function; $u_{2}(0)=0$ and linearity of $c_{2}$ are without loss of generality; while $c_{1}^{\prime}(\infty)=\infty$ ensures that the real balance is bounded above.

I prove Proposition 1 by constructing a mapping whose fixed point $(\rho, \mu)$ is a Definition- 1 steady state. As given in the appendix, this mapping assigns

\footnotetext{
${ }^{4}$ As is explained below, the young need not leave the centralized marekt with the same amount of money.
} 
to each $(\rho, \mu)$ the set of probability measures implied by all randomizations over $X(\rho, \rho, \mu)$, and assigns some $\rho^{\prime}$ to each measure $\sigma$ in this set such that $\rho^{\prime} \gtrless \rho$ if $\int x \sigma(d x) \gtrless 1$. That is, the mapping raises (reduces) the real balance if the excess demand for money is positive (negative).

To carry out this approach, I must find a lower bound on $\rho$ so that the excess demand for money is positive if $\rho$ is close to this bound. In turn, I must show that for small $\rho$, the additional payoff to a buyer in a pairwise meeting from raising his pre-meeting state from $x \leq 1$ to $x+\epsilon$ is sufficiently large. This uses $u_{2}^{\prime}(0)=\infty$ and boundedness of $h$; I need to bound $h$ because the best lower bound I can find on the buyer's additional payoffs from the $\epsilon$ change in the pre-meeting state is $[a+h(x \rho)]^{-1} u_{2}^{\prime}(x \rho) u_{3}^{\prime}(x \rho) \rho \epsilon$ for some constant $a$.

For comparison and for future reference, consider the model without pairwise meetings; that is, the special case, $u_{2}=c_{2}=0$. Then, we have

Corollary 1 If $u_{2}=c_{2}=0$, then $(1+\tau) c_{1}^{\prime}(0)<u_{3}^{\prime}(0)$ is sufficient and necessary for existence of a monetary steady state.

Proof. Let $(\rho, \mu)$ be a Definition-1 steady state with $u_{2}=c_{2}=0$. Under the maintained assumptions, $\mu=\mu_{d}$, where $\mu_{d}$ is the degenerate distribution whose support is $\{1\}$, and $\rho$ is determined by $(1+\tau) c_{1}^{\prime}(\rho)=u_{3}^{\prime}(\rho)$. Because $c_{1}^{\prime \prime}>0,(1+\tau) c_{1}^{\prime}(0)<u_{3}^{\prime}(0) \Leftrightarrow \rho>0$.

\section{The optimal rate of money transfer}

Here, optimality is maximization of the young person's steady-state expected lifetime utility, golden rule optimality. I give sufficient condition conditions for a positive transfer to be optimal.

Proposition 2 If $u_{3}^{\prime}(q) q$ is non decreasing and $u_{3}^{\prime \prime}<0$ and if $\theta$ is sufficiently close to 1 , then the golden-rule rate of transfer is positive.

Proof. The proof proceeds in two steps. In step 1, I prove the result for $\theta=1$; a useful intermediate result is uniqueness of the steady state under each transfer rate $\tau$. In step 2, I show that the result holds for $\theta$ near 1 ; here, I use the uniqueness result in step 1 and a continuity property of the main mapping in the proof of Proposition 1.

Step 1. I first characterize the Definition 1 steady state when $\theta=1$ without appealing to monotonicity of $u_{3}^{\prime}(q) q$ and $u_{3}^{\prime \prime}<0$. 
Fix $\tau$ and a steady state $(\rho, \mu)$. Since $\theta=1$, the steady-state value function $v$ (see (4)) is strictly concave, so $\mu=\mu_{d}$, where $\mu_{d}$ is the degenerate distribution whose support is $\{1\}$. To determine $\rho$, first let the trade in the meeting between a buyer with $x>0$ and a seller with $1,(q(x, 1 ; \rho), l(x, 1 ; \rho))$ (see (1)). By $\theta=1, c_{2}(q)=q$ and $u_{2}^{\prime}(0)=\infty$,

$$
q(x, 1 ; \rho)=u_{3}(\rho+l(x, 1 ; \rho) \rho)-u_{3}(\rho),
$$

and

$$
u_{2}^{\prime}(q(\rho)) u_{3}^{\prime}(\rho+l(x, 1 ; \rho) \rho) \geq u_{3}^{\prime}(x \rho-l(x, 1 ; \rho) \rho) \text { strict only if } l(x, 1 ; \rho)=x .
$$

So the buyer's payoff $f(x, 1 ; \rho)$ (see $(2))$ is $u_{2}(q(x, 1 ; \rho))+u_{3}(\rho-l(x, 1 ; \rho) \rho)$ and when $x=1$, a marginal increment of the buyer's pre-meeting state increases his payoff by $u_{2}^{\prime}(q(\rho)) u_{3}^{\prime}(\rho+l(\rho) \rho) \rho$, where $(q(\rho), l(\rho))=(q(x, 1 ; \rho), l(x, 1 ; \rho))$. Next, in the meeting between a seller with $x>0$ and a buyer with 1 , by $\theta=1$, the seller's payoff $g(1, x ; \rho)$ (see $(3))$ is $u_{3}(x \rho)$ and when $x=1$, a marginal increment of the seller's pre-meeting state increases his payoff by $u_{3}^{\prime}(\rho) \rho$. Then by market clearing, $\rho$ must satisfy $F(\rho, \tau)=0$, where

$$
F(\rho, \tau)=(1+\tau) c_{1}^{\prime}(\rho)-0.5 u_{2}^{\prime}(q(\rho)) u_{3}^{\prime}(\rho+l(\rho) \rho)-0.5 u_{3}^{\prime}(\rho) .
$$

The young person's expected lifetime utility in this steady state is

$$
W(\rho)=-c_{1}(\rho)+0.5 u_{2}(q(\rho))+0.5 u_{3}(\rho-l(\rho) \rho)+0.5 u_{3}(\rho) .
$$

Next, I show a weaker result: If $c_{1}^{\prime}(0) \geq u_{3}^{\prime}(0)$, some positive transfer dominates zero transfer. That is, when the pairwise meetings are necessary for money to be valued at $\tau=0$ (see Corollary 1), some positive transfer is beneficial.

To see this result, first fix $\tau \geq 0$. Since $c_{1}^{\prime}(0) \geq u_{3}^{\prime}(0), F(\rho, \tau)=0$ only if the inequality in (8) with $x=1$ is strict. So $l(\rho)=1$ is necessary for $F(\rho, \tau)=0$. Fixing $l(\rho)=1$ in $(9)$, then $\forall \rho>0, F_{1}(\rho, \tau)$ (the derivative of $F(, \tau)$ at $\rho$ ) is defined and positive. So there exists a unique $\rho$, denoted $\rho(\tau)$, satisfying $F(\rho, \tau)=0$. Next, by the implicit function theorem (applied to $F(\rho(\tau), \tau)=0), \forall \tau \geq 0, \rho^{\prime}(\tau)=-c_{1}^{\prime}(\rho(\tau)) / F_{1}(\rho(\tau), \tau)<0$. Next, fixing $l(\rho)=1$ in (10) and using $F(\rho(\tau), \tau)=0$, we obtain the derivative of $W$ at $\rho(\tau)$ as

$$
W^{\prime}(\rho)=\tau c_{1}^{\prime}(\rho)+0.5 u_{2}^{\prime}(q) \Delta
$$


where $\rho=\rho(\tau)$ and

$$
\Delta=u_{3}^{\prime}(2 \rho)-u_{3}^{\prime}(\rho) .
$$

Finally, let $V(\tau)=W(\rho(\tau))$ so

$$
V^{\prime}(0)=W^{\prime}(\rho(0)) \rho^{\prime}(0) .
$$

By $u_{3}^{\prime \prime}<0, \Delta<0$ so $W^{\prime}(\rho(0))<0$. Then by $\rho^{\prime}(0)<0, V^{\prime}(0)>0$. This proves the weaker result.

To extend the weaker result to the one in the proposition, I need to show $V$ is strictly increasing on $(-1, \bar{\tau})$ for some $\bar{\tau}>0$ without appealing to $c_{1}^{\prime}(0) \geq u_{3}^{\prime}(0)$. The argument is similar, but I need to deal with a technical issue ruled out by $c_{1}^{\prime}(0) \geq u_{3}^{\prime}(0)$ and $\tau \geq 0$ : it may be the case that in a steady state $\left(\rho, \mu_{d}\right)$ under some $\tau, l(\rho)=1$ and (8) with $x=1$ holds with equality (the buyer's cash constraint just binds), so that $l^{\prime}(\rho), W^{\prime}(\rho)$ and $\rho^{\prime}(\tau)$ may not be defined.

As is shown in Lemma 4 in the appendix, $\forall \tau>-1$, there exists a unique $\rho$, denoted $\rho(\tau)$, satisfying $F(\rho, \tau)=0$; the derivative $W$ at $\rho(\tau)$ is determined by

$$
W^{\prime}(\rho)=\tau c_{1}^{\prime}(\rho)+0.5 u_{2}^{\prime}(q) \Delta
$$

where $\rho=\rho(\tau)$ and

$$
\Delta=u_{3}^{\prime}(\rho+l(\rho) \rho)-u_{3}^{\prime}(\rho)
$$

and the right and left derivatives of $\rho($.$) at \tau$ are defined and negative. Again, $u_{3}^{\prime \prime}<0 \Rightarrow \Delta<0$, so the golden rule rate of transfer is positive. This completes step 1.

Step 2. See Lemma 5 in the appendix.

By the definition of $W^{\prime}$ (see (12)), if the real balance changes by $d \rho$ in the steady state $\left(\rho(\tau), \mu_{d}\right)$, the young person's expected lifetime utility changes by $W^{\prime}(\rho(\tau)) d \rho$. Because $W^{\prime}(\rho(\tau)) \neq 0$, there are effects from the change in the real balance the young person can not capture by his own action-in specific, by choosing his own saving in term of money (notice that if a young person changes real saving by $d \rho$, his expected lifetime utility changes by $F(\rho(\tau), \tau) d \rho)$. In $(12), \tau c_{1}^{\prime}(\rho)$ reflects one such effect: Each young person's real wealth changes by $\tau d \rho$ in a lump sum way. Also, $u_{2}^{\prime}(q(\rho)) \Delta$ reflects another such effect: For a young person who is a buyer in pairwise meeting, if his payment measured by its real value in the coming competitive market does not change, the amount of good he receives from the seller changes by 
$\Delta d \rho$. The first effect presents in ordinary OLG models; the second does not. The second effect comes from the externality described in the introduction.

There is no such externality when the old are risk neutral, so a positive transfer cannot dominate zero transfer.

Corollary 2 If $u_{3}^{\prime \prime}=0$ and if $\theta$ is sufficiently close to 1 , then the golden-rule rate of transfer is non positive.

Proof. When $\theta=1, u_{3}^{\prime \prime}=0$ implies $\Delta=0$ in (12). So the golden-rule rate of transfer is zero. The rest of the proof follows the exact argument in Lemma 5.

I suspect that Corollary 2 holds for general $\theta<1$, but proving such result is difficult because the distribution $\mu$ in a steady state need not be degenerate. With more restrictive assumptions to ensure such degeneracy for general $\theta$, I can show that $\rho(\tau)$ is strictly decreasing in $\tau$ and $W^{\prime}(\rho(0))$ has the same sign as

$$
\eta \equiv(1-\theta)+(1-\theta) u_{2}^{\prime}(q(1,1 ; \rho))+\theta u_{2}^{\prime \prime}(q(1,1 ; \rho))[q(1,1 ; \rho)-\rho],
$$

which implies some negative transfer dominates zero transfer $(\theta<1 \Rightarrow$ $q(1,1 ; \rho)<\rho \Rightarrow \eta>0)$. The term $\eta$ reflects the so-called holdup problem. This problem does not rely on the curvature of $u_{3}$, and it can be dominant when the buyer's bargaining power is far away from unity so that some negative transfer dominates zero transfer even if the old are risk averse.

When pairwsie meetings are dropped, both the externality driving Proposition 2 and the holdup problem are absent and there is no room for policy intervention.

Corollary 3 If $u_{2}=c_{2}=0$, then the golden-rule rate of money transfer is zero.

Proof. If $c_{1}^{\prime}(0) \geq u_{3}^{\prime}(0)$, then $\rho>0$ only if $\tau<0$ (see Corollary $1)$. But the young person's expected steady state utility is less than $a=$ $-c_{1}(0)+u_{3}(0)$. If $c_{1}^{\prime}(0)<u_{3}^{\prime}(0)$ and if $(1+\tau) c_{1}^{\prime}(\rho)=u_{3}^{\prime}(\rho)$ has a positive solution, denoted $\rho(\tau)$, then the person's expected lifetime utility is $W(\rho(\tau))=$ $-c_{1}(\rho(\tau))+u_{3}(\rho(\tau))$ (notice that $W(\rho(0))>a$ ). Because $W^{\prime}(\rho(\tau))=$ $\tau c_{1}^{\prime}(\rho(\tau))$ and $\rho^{\prime}(\tau)<0$, the golden-rule rate is zero.

Policy is irrelevant if the real balance does not respond to it. 
Corollary 4 If $c_{1}(x)=0$ for $x \in[0, C]$, then the young person's steady-state expected lifetime utility does not depend on $\tau$.

Proof. Fix $\theta$. It suffices to note that under each $\tau$, there exists a unique steady state $(\rho, \mu)=\left(C, \mu_{d}\right)$.

Thus far, I consider transfers to the young in the centralized market. Such transfers and transfers to the old are equivalent if buyers' cash constraints are not binding in pairwise meetings. Otherwise, the two types of transfers support different allocations and the optimality of a positive transfer to the young does not imply the optimality of a positive transfer to the old. For example, in a Corollary-4 setting with $C=1, u_{2}(q)=2 \sqrt{q}$, and $u_{3}(q)=q$, all transfers to the young are equivalent, but any positive transfer to the old decreases welfare of the young. A related point is the risk-sharing effect of money creation. Levine [7] presents an infinitely-lived agents model in which money creation is beneficial because of this effect. Pairwise trade in this OLG model does induce a risk to each young person, but, by itself, the risk does not call for policy intervention. Indeed, in any Corollary-4 setting, a positive transfer to the old gives rise to risk-sharing, but such a transfer reduces the young person's steady-state expected lifetime utility.

Finally, even if the pairwise trading protocol is price taking, a positive transfer to the young can still be beneficial. With some additional assumptions, I can show $\rho^{\prime}(\tau)<0, W^{\prime}(\rho(0))$ has the same sign as $(\kappa / \rho)^{\prime}$, where $\kappa$ is the stage-2 real balance. Moreover, $(\kappa / \rho)^{\prime}<0$ if $u_{3}^{\prime \prime}<0$, and $(\kappa / \rho)^{\prime}=0$ if $u_{3}^{\prime \prime}=0$. So some positive transfer dominates zero transfer if $u_{3}^{\prime \prime}<0$. To see why $(\kappa / \rho)^{\prime}$ matters, consider a buyer with state 1 in stage 2 who chooses $q_{b 2}$ (stage-2 consumption) and $q_{b 3}$ (stage- 3 consumption) to maximize

$$
u_{2}\left(q_{b 2}\right)+u_{3}\left(q_{b 3}\right) \text {, s.t. }(\kappa / \rho) q_{b 3}+q_{b 2}=\kappa \text { and } q_{b 2} \leq \kappa .
$$

Now a change in $\rho$ has two two effects on his first constraint - one through a change in $\kappa$ and the other through a change in $(\kappa / \rho)$, and the second effect is the one that he can not capture by his own action.

\section{Some variants of the model}

There are several variants of the basic model. In one variant, the old can produce and the young can consume in the centralized market. The old's disutility from producing $q$ is $c_{3}(q)$, and the young's utility from consuming 
$q$ is $u_{1}(q)$. Let $c_{3}$ be strictly increasing, strictly convex and differentiable with $c^{\prime}(0)=0$. Let $u_{1}$ be strictly increasing and concave.

Now an old person in state $x$ will receive the payoff

$$
w\left(x ; \rho_{t+1}\right)=\max _{q \geq x \rho_{t}} u_{3}(q)-c_{3}\left(q-x \rho_{t+1}\right) .
$$

Replacing $u_{3}\left(a \rho_{t+1}\right)$ by $w\left(a ; \rho_{t+1}\right)$ in (1)-(3), revising the objective function in (5) as $u_{1}(q)-c_{1}\left(q+l \rho_{t}\right)+v\left(\frac{l+\tau}{1+\tau}\right)$, and revising the budget constraint in (5) as $q \geq 0$ and $l \geq \max \{0,-\tau\}$, the definition of equilibrium goes through.

Some useful properties of $w$ are summarized in the next lemma.

Lemma 1 (i) $\forall \rho>0, x \mapsto w(x ; \rho)$ is strictly increasing, concave, and twice differentiable. Also, $x \mapsto q_{3}(x ; \rho)$ is strictly increasing. (ii) $\forall x>0$, $\rho \mapsto w(x ; \rho)$ is strictly increasing, concave, and twice differentiable. Also, $\rho \mapsto q_{3}(x ; \rho)$ is strictly increasing. (iii) The derivative of $w(. ; \rho)$ at $x$ and the derivative of $w(x ;$.$) at \rho$ are

$$
w_{1}(x ; \rho)=u_{3}^{\prime}\left[q_{3}(x ; \rho)\right] \rho \text { and } w_{2}(x ; \rho)=u_{3}^{\prime}\left[q_{3}(x ; \rho)\right] x,
$$

respectively.

Proof. Monotonicity and concavity in the lemma are standard. By $c_{3}^{\prime}(0)=0, q_{3}(x ; \rho)>x \rho$ and $c_{3}^{\prime}\left[q_{3}(x ; \rho)-x \rho\right]=u_{3}^{\prime}\left[q_{3}(x ; \rho)\right]$. Then by $c_{3}^{\prime \prime}>0$ and the implicit function theorem, $x \mapsto q_{3}(x ; \rho)$ and $\rho \mapsto q_{3}(x ; \rho)$ are twice differentiable. This and the envelop theorem imply the rest of the lemma.

The following is analogous to Proposition 2.

Proposition 3 If $u_{3}^{\prime}(q) q$ is non decreasing and $w(. ; \rho)$ is strictly concave and if $\theta$ is sufficiently close to 1 , then the golden-rule rate of transfer is positive.

In the proof of this proposition, the analogy to (12) is

$$
W^{\prime}(\rho)=\tau c_{1}^{\prime}(\rho)+0.5 u_{2}^{\prime}(q) \Delta
$$

where $\rho=\rho(\tau)$ and

$$
\Delta=w_{1}(1+l(\rho) ; \rho)-w_{1}(1 ; \rho) .
$$

The following is analogous to Corollary 2. 
Corollary 5 If $u_{3}^{\prime}(q) q$ is non decreasing and $w(. ; \rho)$ is affine and if $\theta$ is sufficiently close to 1 , then the golden-rule rate of transfer is non positive.

One can revise this variant by assuming that there is some probability that a person can not produce at his third stage. Also, one can assume that there is some probability that a person is idle in his second stage. The analogies to the above proposition and corollary still hold. In specific, the strict curvature of the post-pairwise-trade value function of money is necessary for positive transfer to be optimal.

\section{Discussions}

There is no capital in the present model. One may introduce capital as an input for production in the centralized market. To maintain the transactions role of money, one may assume that neither capital nor certificates of its ownership can be carried into pairwise meetings. ${ }^{5}$ More interestingly, one could assume some private information about the quality of capital, similar to the private information on the quality of goods in Williamson and Wright [15]. Neither approach would eliminate the externality driving Proposition 2. Of course, in a version with capital, money creation could induce more capital accumulation, an effect that has long been discussed in literature (see, e.g., Tobin [13]).

Molico [11] studies a search model with infinitely-lived agents and with only pairwise trade. He finds examples in which money creation is beneficial. Because he assumes take-it-or-leave-it offers by buyers, some version of the externality driving Proposition 2 shall attribute to that. Deviatov and Wallace [6] and Deviatov [5] study a version of the model in [11]. They select a trading protocol to maximize a social planner's objective function. They find examples in which money creation is beneficial. Results from $[5,6]$ motivate the following question: In the OLG model, if the government can choose the rate of money transfer and the trading protocol (subject to some participation constraints), does the optimal policy involve money creation?

Lagos and Wright [8] (LW) build an infinitely-lived agent model with alternating decentralized-centralized markets. For tractability, they make a special assumption that implies the post-pairwise-meeting value function of

\footnotetext{
${ }^{5}$ Aruoba and Wright [3] make such an assumption. As long as olds are risk averse, one can break dichotomy reported by [3] in the OLG model.
} 
money is affine. They find that the Friedman rule is optimal. This OLG model was initially motived to understand significance of wealth effects of money creation that are eliminated by that special assumption. The start of thinking is a sort of observational equivalence between the LW model and the OLG model with risk neutral the old. Although the representative agent's steady-state expected discount utility in the LW model, and the young person's steady-state expected lifetime utility in the equivalent OLG model are maximized by different rates of money transfer, the difference disappears if people in the LW model are not endowed with the initial money stock (see the appendix for more details). Based on these findings and Proposition 2, I suspect that in the LW model with a strictly concave post-pairwise-meeting value function of money, if the buyer's bargaining power is close to unity, the Friedman rule is not optimal. ${ }^{6}$

This OLG model is tractable because people only live for three stages. Such a model is not suitable for serious quantitative exercises, but it has a natural extension for such exercises. One version would have, an old person dies with some probability. Of course, then, the inherited distribution of wealth would become a state variable. Tractability would be lost in such a version model, so it would have to be solved numerically.

\section{Appendix}

\section{Proof of Proposition 1}

As is indicated in the main text, I shall define a mapping whose fixed point is a Definition-1 steady state, and the main issue is to find a lower bound on $\rho$ that can be preserved by the mapping. When $u_{3}$ is not bounded below, it is fairly obvious to find such a lower bound, but the mapping differs in some detail from the mapping when $u_{3}$ is bounded below. To focus on the main issue, here I deal with $u_{3}$ bounded below.

Let $\rho_{0}>0$ and $\rho_{1}>\rho_{0}$, and let $Z>1$. Let $\boldsymbol{\rho}=\left[\rho_{0}, \rho_{1}\right]$, and let $\boldsymbol{\mu}$ be the set of all probability measures on $[0, Z]$. For $\mu \in \boldsymbol{\mu}$, let $E_{\mu} \equiv \int x \mu(d x)$, and let the measure $\bar{\mu}$ be defined by $\bar{\mu}(A) E_{\mu}=\mu(A)$ if $E_{\mu}>1$, and $\bar{\mu}(A)=\mu(A)$ if $E_{\mu} \leq 1$. For $(\rho, \mu) \in \boldsymbol{\rho} \times \boldsymbol{\mu}$, let $\Sigma(\rho, \mu)$ be the set of probability measures

\footnotetext{
${ }^{6} \mathrm{~A}$ recent paper by Chiu and Molico [4] deal with money creation in the LW model without the special assumption. Their numerical findings seem to be consistent with this conjecture.
} 
implied by all randomizations over $X(\rho, \rho, \bar{\mu})$. Then let

$$
\begin{aligned}
& T_{1}(\rho, \mu)=\left\{\rho+\rho_{0}\left(E_{\sigma}-1\right) / Z: \sigma \in \Sigma(\rho, \mu)\right\}, \\
& T_{2}(\rho, \mu)=\Sigma(\rho, \mu) .
\end{aligned}
$$

A fixed point of $T=T_{1} \times T_{2}$ is a Definition-1 steady state.

The next lemma provides conditions about $\left(\rho_{0}, \rho_{1}, Z\right)$ under which there exists a fixed point of $T$.

Lemma 2 Let $\rho_{0} \in(0,1), \rho_{1}>3$, and $Z>1$. Suppose for $(\rho, \mu) \in \boldsymbol{\rho} \times \boldsymbol{\mu}$, (R1) $\rho>\rho_{1}-\rho_{0} \Rightarrow \max X(\rho, \rho, \bar{\mu}) \leq 1$; (R2) $\rho<2 \rho_{0} \Rightarrow \min X(\rho, \rho, \bar{\mu}) \geq 1$; and $(R 3) \max X(\rho, \rho, \bar{\mu}) \leq Z$. Then there exists a fixed point of $T=T_{1} \times T_{2}$.

Proof. First, fix $(\rho, \mu) \in \boldsymbol{\rho} \times \boldsymbol{\mu}$ and $\sigma \in \Sigma(\rho, \mu)$. By (R3), $E_{\sigma} \leq Z$, so $\left(E_{\sigma}-1\right) / Z \in(-1,1)$. Let $\rho^{\prime}=\rho+\rho_{0}\left(E_{\sigma}-1\right) / Z$. If $\rho \in\left[2 \rho_{0}, \rho_{1}-\rho_{0}\right], \rho^{\prime} \in \boldsymbol{\rho}$ immediately. If $\rho>\rho_{1}-\rho_{0}$, (R1) $\Rightarrow E_{\sigma} \leq 1 \Rightarrow \rho^{\prime} \in \boldsymbol{\rho}$. If $\rho<2 \rho_{0}$, (R2) $\Rightarrow$ $E_{\sigma} \geq 1 \Rightarrow \rho^{\prime} \in \boldsymbol{\rho}$. So $T_{1}(\rho, \mu) \subset \boldsymbol{\rho}$. Then by (R3), $T(\rho, \mu) \subset \boldsymbol{\rho} \times \boldsymbol{\mu}$.

Next, by $u_{3}^{\prime \prime} \leq 0$ and $u_{2}^{\prime \prime}<0,(q(x, y ; \rho), l(x, y ; \rho))$ (see $\left.(1)\right)$ is the unique $(q, l)$ satisfying

$$
\begin{aligned}
& u_{2}^{\prime}(q) u_{3}^{\prime}(y \rho+l \rho) \geq u_{3}^{\prime}(x \rho-l \rho) \text { strict only if } l=x, \\
& \theta u_{2}^{\prime}(q)\left[-q+u_{3}(y \rho+l \rho)-u_{3}(y \rho)\right] \\
= & (1-\theta)\left[u_{2}(q)+u_{3}(x \rho-l \rho)-u_{3}(x \rho)\right] .
\end{aligned}
$$

By the theorem of maximum, $(x, y, \rho) \mapsto q(x, y ; \rho)$ and $(x, y, \rho) \mapsto l(x, y ; \rho)$ are continuous, so are $(x, y, \rho) \mapsto f(x, y ; \rho)$ and $(x, y, \rho) \mapsto g(x, y ; \rho)$.

Next, let $\boldsymbol{\mu}$ be equipped with the weak* topology, and let $\mathbf{V}$ be the set of real-valued continuous functions on $[0, Z]$ equipped with the sup norm topology. Since $\mu \mapsto \bar{\mu}$ is continuous, it follows from [2, 14.7 Corollary, p. 480] and continuity of $(x, y, \rho) \mapsto f(x, y ; \rho)$ and $(x, y, \rho) \mapsto g(x, y ; \rho)$ that $(\rho, \mu) \mapsto v(. ; \rho, \bar{\mu})$ is continuous. Then by the theorem of maximum, $(\rho, \mu) \mapsto X(\rho, \rho, \bar{\mu})$ is u.h.c. and compact-valued. Hence $(\rho, \mu) \mapsto \Sigma(\rho, \mu)$ is u.h.c., compact-valued, and convex-valued, and so is $(\rho, \mu) \mapsto T(\rho, \mu)$. By Fan's fixed point theorem, there exists a fixed point of $T$.

The next lemma completes the proof.

Lemma 3 There exist $\rho_{0} \in(0,1), \rho_{1}>3$, and $Z>1$ satisfying (R1)-(R3) in Lemma 2. 
Proof. The proof proceeds by three steps. In step 1 , I show that there exists $\rho_{1}>3$ satisfying (R1); in step 2, I show that there exists $\rho_{0} \in(0,1)$ satisfying (R2); in step 3 , I show that given $\left(\rho_{0}, \rho_{1}\right)$, there exists $Z>1$ satisfying (R3). Inside the proof, I suppress dependence of $q(x, y ; \rho), l(x, y ; \rho)$, $f(x, y ; \rho), g(x, y ; \rho)$, and $v(x ; \rho, \mu)$ on $(\rho, \mu)$.

Step 1 . Let $\tilde{\rho}>2$ satisfy $u_{2}^{\prime}(d)<1$, where $u_{2}(d)=u_{3}(\tilde{\rho})-u_{3}(1)$, and

$$
(1+\tau) c_{1}^{\prime}(\tilde{\rho})>u_{3}^{\prime}(1) .
$$

Then let $\rho_{1}>\tilde{\rho}+1$. Fix $\mu, \rho>\rho_{1}-1$ (so $\left.\rho>\tilde{\rho}\right)$ and $z>1$. It is standard to show that $u_{2}^{\prime \prime}<0, u_{3}^{\prime} \leq 0$, (17), and (18) imply $g(y, z)-g(y, z-\epsilon) \leq$ $u_{3}^{\prime}(z \rho-\epsilon \rho) \epsilon \rho, \forall \epsilon \in(0, z)$ and $y$. Now I claim $f(z, y)-f(z-\epsilon, y) \leq u_{3}^{\prime}(1) \epsilon \rho, \forall$ $\epsilon \in(0, z-1)$ and $y$. Therefore, $u_{3}^{\prime}(1) \epsilon \rho \geq v(z)-v(z-\epsilon)$ for small $\epsilon$. Then by $d=[(1+\tau) z-\tau] \rho>\tilde{\rho}$ and $(19), v(z)-v(z-\epsilon)<c_{1}(d)-c_{1}(d-(1+\tau) \epsilon \rho)$ for small $\epsilon$, so $z \notin X(\rho, \rho, \bar{\mu})$ and hence $\max X(\rho, \rho, \bar{\mu}) \leq 1$.

For the claim, it suffices to show that $f_{1}(x, y)$ (the derivative of $f(., y)$ at $x$ ) is bounded above by $u_{3}^{\prime}(1) \rho, \forall x>1$ and $y$. Fix $x>1$ and $y$, and let $(\kappa, \iota)=(q(x, y), l(x, y))$. Suppose $x \rho-\iota \rho<1$. Then by $x \rho>\tilde{\rho}$ and $u_{2}(\kappa)>u_{3}(x \rho)-u_{3}(x \rho-\iota \rho), \kappa>d$ so $u^{\prime}(\kappa)<1$. Then by $(17), \iota<0.5 x$ so $0.5 x \rho<x \rho-\iota \rho<1$ or $x \rho<2$, which contradicts $\tilde{\rho}>2$. So $x \rho-\iota \rho>1$ and (17) holds with equality. Then by the implicit function theorem (applied to (17) with equality and (18)), $q_{1}(x, y)$ (the derivative of $q(., y)$ at $x$ ) and $l_{1}(x, y)$ (the derivative of $l(., y)$ at $\left.x\right)$ are defined, in particular,

$$
q_{1}=\frac{\theta u_{2}^{\prime}(\kappa) u_{3}^{\prime}(y \rho+\iota \rho) l_{1}-(1-\theta) u_{3}^{\prime}(x \rho-\iota \rho)\left(1-l_{1}\right)+(1-\theta) u_{3}^{\prime}(x \rho)}{u_{2}^{\prime}(\kappa)-(1-\theta)\left[u_{2}(\kappa)+u_{3}(x \rho-\iota \rho)-u_{3}(x \rho)\right] u_{2}^{\prime \prime}(\kappa) / u_{2}^{\prime}(\kappa)} \rho .
$$

Now $f_{1}(x, y)$ is defined and is determined by

$$
f_{1}=u_{2}^{\prime}(\kappa) q_{1}+u_{3}^{\prime}(x \rho-\iota \rho)\left(1-l_{1}\right) \rho .
$$

It can be shown that $l_{1} \leq 1$. Then by $x \rho-\iota \rho>1, f_{1}(x, y) \leq u_{3}^{\prime}(1) \rho$.

Step 2. Let $\alpha>0$ and $\hat{q}>0$ satisfy $1+1 / \theta+h(q)<\alpha, \forall q \leq \hat{q}$. Let $\bar{\rho} \in(0,1)$ satisfy $\bar{q}=u_{3}(\bar{\rho})-u_{3}(0) \leq \hat{q}$,

$$
u_{2}^{\prime}(\bar{q}) u_{3}^{\prime}(3 \bar{\rho})>4 \alpha(1+\tau) c_{1}^{\prime}(\bar{\rho}),
$$

and $u_{2}^{\prime}(\bar{q}) u_{3}^{\prime}(3 \bar{\rho})>u_{3}^{\prime}(0)$ if $u_{3}^{\prime}(0)<\infty$. Then let $\rho_{0} \in(0,0.5 \bar{\rho})$. Fix $\mu$, $\rho\left(0,2 \rho_{0}\right)$ (so $\left.\rho<\bar{\rho}\right)$ and $z<1$. It is standard to show that $u_{2}^{\prime \prime}<0, u_{3}^{\prime} \leq 0$, (17), and (18) imply $f(z+\epsilon, y)>f(z, y)$ and $g(y, z+\epsilon)>g(y, z), \forall \epsilon>0$ 
and $y$. Now I claim $\alpha[f(z+\epsilon, y)-f(z, y)]>u_{2}^{\prime}(\bar{q}) u_{3}^{\prime}(3 \bar{\rho}) \epsilon \rho, \forall \epsilon \in(0,1-z)$ and $y$. Therefore, by $\bar{\mu}\{y: y \leq 2\} \geq 1 / 2,4 \alpha[v(z+\epsilon)-v(z)]>u_{2}^{\prime}(\bar{q}) u_{3}^{\prime}(3 \bar{\rho}) \epsilon \rho$ for small $\epsilon$. Then by $d=[(1+\tau) z-\tau] \rho<\bar{\rho}$ and $(22), v(z+\epsilon)-v(z)>c_{1}(d+$ $\epsilon(1+\tau) \rho)-c_{1}(d)$ for small $\epsilon$, so $z \notin X(\rho, \rho, \bar{\mu})$ and hence $\min X(\rho, \rho, \bar{\mu}) \geq 1$.

For the claim, it suffices to show that $f_{1}(x, y)$ is bounded below by $u_{2}^{\prime}(\bar{q}) u_{3}^{\prime}(3 \bar{\rho}) \rho, \forall x<1$ and $y \leq 2$. Fix $x<1$ and $y \leq 2$, and let $(\kappa, \iota)=$ $(q(x, y), l(x, y))$. If $u_{3}^{\prime}(0)=\infty$, by the implicit function theorem (applied to (17) with equality and (18)), $q_{1}(x, y)$ and $l_{1}(x, y)$ are defined, and so is $f_{1}(x, y)$. If $u_{3}^{\prime}(0)<\infty$, by $u_{2}^{\prime}(\bar{q}) u_{3}^{\prime}(3 \bar{\rho})>u_{3}^{\prime}(0)$ and $\rho<\bar{\rho}, l(x, y)=x$ so $l_{1}(x, y)=1$; also, $q(x, y)$ is the $q$ satisfying (18) with $l=x$, and by the implicit function theorem, $q_{1}(x, y)$ is defined and so is $f_{1}(x, y)$. Either way, $q_{1}(x, y)$ is determined by $(20)$, and $f_{1}(x, y)$ is determined by $(21)$. Then by $q(x, y)<\bar{q} \leq \hat{q}, \alpha f_{1}(x, y)>u_{2}^{\prime}(\bar{q}) u_{3}^{\prime}(3 \bar{\rho}) \rho$.

Step 3. Given $\rho_{0}$, let $Z>1$ satisfy

$$
(1+\tau) c_{1}^{\prime}\left((1+\tau) Z \rho_{0}-\tau \rho_{0}\right)>u_{3}^{\prime}(1) .
$$

Fix $\mu, \rho \geq \rho_{0}$ and $z>Z$. By the argument in step 1, I can show $z \notin$ $X(\rho, \rho, \bar{\mu})$, so $\max X(\rho, \rho, \bar{\mu}) \leq Z$.

\section{Completion of the proof of Proposition 2}

The next lemma completes the proof of step 1.

Lemma 4 (i) $\forall \tau>-1$, there exists a unique $\rho$, denoted $\rho(\tau)$, satisfying $F(\rho, \tau)=0$. (ii) $\rho():.(-1, \infty) \rightarrow \mathbb{R}$ is strictly decreasing. (iii) The left and right derivatives of $\rho($.$) at any \tau$ are defined. (iv) The derivative of $W$ at $\rho(\tau)$ is $W^{\prime}(\rho)=\tau c_{1}^{\prime}(\rho)+0.5 u_{2}^{\prime}(q(\rho)) \Delta$, where $\rho=\rho(\tau)$ and $\Delta=$ $u_{3}^{\prime}(\rho+l(\rho) \rho)-u_{3}^{\prime}(\rho)$.

Proof. We first summarize preliminary results into five claims.

Claim 1: There exists a unique $\hat{\rho}$ satisfying

$$
u_{2}^{\prime}\left(u_{3}(2 \hat{\rho})-u_{3}(\hat{\rho})\right) u_{3}^{\prime}(2 \hat{\rho})=u_{3}^{\prime}(0) .
$$

To see this, first by monotonicity of $u_{3}^{\prime}(q) q, 2 u_{3}^{\prime}(2 \rho)-u_{3}^{\prime}(\rho) \geq 0$. Then the result follows from $u_{2}^{\prime}(0)=\infty$ and $u_{3}^{\prime \prime}<0$. (It is worth noting that claim 1 holds when $u_{3}^{\prime \prime}=0$.)

Claim 2: If $\rho \leq \hat{\rho}, l(\rho)=1$. To see this, first note that $l(\hat{\rho})=1$. Next let $\rho<\hat{\rho}$. By monotonicity of $u_{3}^{\prime}(q) q, u_{3}(2 \rho)-u_{3}(\rho) \leq u_{3}(2 \hat{\rho})-u_{3}(\hat{\rho})$. If $l(\rho)<1$, then (8) with $x=1$ holds with equality, which contradicts $(23)$. 
Claim 3: If $\rho>\hat{\rho}, l(\rho)<1$ and (8) with $x=1$ holds with equality. To see this, notice that $u_{2}^{\prime}\left(u_{3}(2 \rho)-u_{3}(\rho)\right) u_{3}^{\prime}(2 \rho)>u_{3}^{\prime}(0)$ and $\rho>\hat{\rho}$ contradict (23).

Claim 4: $l^{\prime}(\rho)$ is defined at $\rho \neq \hat{\rho}$; also, $l_{-}(\hat{\rho})$ (the left derivative of $l($.$) at$ $\hat{\rho}$ ) and $l_{+}^{\prime}(\hat{\rho})$ (the right derivative of $l($.$) at \hat{\rho}$ ) are defined. To see this, first by claim $2, l^{\prime}($.$) is defined over (0, \hat{\rho}]$, where $l^{\prime}(\hat{\rho})=l_{-}(\hat{\rho})$. Next by claim 3 and the implicit function theorem (applied to (8) with $x=1$ with equality), $l^{\prime}($.$) is defined over [\hat{\rho}, \infty)$, where $l^{\prime}(\hat{\rho})=l_{+}(\hat{\rho})$.

Claim 5: $\forall \tau>-1, F_{1}(\rho, \tau)$ is defined and positive at $\rho \neq \hat{\rho}$; also, $F_{1-}(\hat{\rho}, \tau)$ (the left derivative of $F(., \tau)$ at $\left.\hat{\rho}\right)$ and $F_{1+}(\hat{\rho}, \tau)$ (the right derivative of $F(., \tau)$ at $\hat{\rho})$ are defined and positive. To see this, fix $\tau$. First by claims 2 and $4, F_{1}(., \tau)$ is defined over $(0, \hat{\rho}]$ and is positive valued, where $F_{1}(\hat{\rho}, \tau)=$ $F_{1-}(\hat{\rho}, \tau)$. Next by claims 3 and $4, F_{1}(., \tau)$ is defined over $[\hat{\rho}, \infty)$, where $F_{1}(\hat{\rho}, \tau)=F_{1+}(\hat{\rho}, \tau)$, and to see $F_{1}(\rho, \tau)>0$, if $l^{\prime}(\rho) \geq 0$, directly differentiate (9) w.r.t. $\rho$; otherwise, first substitute (8) with $x=1$ with equality into (9) and then differentiate it w.r.t. $\rho$.

Now part (i) follows from claim 5. Part (ii) follows from claim 5 and $F(\rho(\tau), \tau)=0$.

For part (iii), first by part (ii), there exists at most one $\hat{\tau}$ satisfying $\rho(\hat{\tau})=\hat{\rho}$. Then by claims 2 and $3, l(\rho(\tau))<1$ if $\tau<\hat{\tau}$, and $l(\rho(\tau))=1$ if $\tau \geq \hat{\tau}$. Now by claim 5 and the implicit function theorem (applied to $F(\rho(\tau), \tau)=0), \rho^{\prime}($.$) is defined over (-1, \hat{\tau}]$, where $\rho^{\prime}(\hat{\tau})=\rho_{-}^{\prime}(\hat{\tau})$ (the left derivative of $\rho($.$) at \hat{\tau})$; also, $\rho^{\prime}($.$) is defined over [\hat{\tau}, \infty)$, where $\rho^{\prime}(\hat{\tau})=\rho_{+}^{\prime}(\hat{\tau})$ (the right derivative of $\rho($.$) at \hat{\tau})$. In specific, $\rho^{\prime}(\tau)=-c_{1}^{\prime}(\rho(\tau)) / F_{1}(\rho(\tau), \tau)$ at $\tau \neq \hat{\tau}, \rho_{-}^{\prime}(\hat{\tau})=-c_{1}^{\prime}(\rho(\tau)) / F_{1+}(\hat{\rho}, \hat{\tau})$, and $\rho_{+}^{\prime}(\hat{\tau})=-c_{1}^{\prime}(\rho(\tau)) / F_{1-}(\hat{\rho}, \hat{\tau})$.

For part (iv), since $l(\rho)=1$ for $\rho \leq \hat{\rho}$, the left derivative of $W$ at $\hat{\rho}$ is $\tau c_{1}^{\prime}(\hat{\rho})+0.5 u_{2}^{\prime}(q(\hat{\rho}))\left[u_{3}^{\prime}(2 \hat{\rho})-u_{3}^{\prime}(\hat{\rho})\right]$. Because (8) with $x=1$ with equality holds for $\rho \geq \hat{\rho}$, the right derivative of $W$ at $\hat{\rho}$ is $\tau c_{1}^{\prime}(\hat{\rho})+0.5 u_{2}^{\prime}(q(\hat{\rho}))\left[u_{3}^{\prime}(\hat{\rho}+\right.$ $\left.l(\hat{\rho}) \hat{\rho})-u_{3}^{\prime}(\hat{\rho})\right]$. Since $l(\hat{\rho})=1$, the two derivatives are equal. Evidently, $W^{\prime}(\rho)$ is defined at $\rho \neq \hat{\rho}$ and takes the purported value.

The next lemma deals with step 2 of the proof.

Lemma 5 There exists some $\bar{\theta}<1$ such that the golden rule rate of transfer is positive if $\theta>\bar{\theta}$.

Proof. First, adding $\theta$ into the lists of arguments of $q(),. l(),. f($.$) ,$ and $g($.$) , we have mappings (x, y, \rho, \theta) \mapsto q(x, y, \rho, \theta)$ and so on. Denoting a 
generic $(x, y, \rho, \theta)$ by $a$, I first show $a \mapsto q(a)$ and $a \mapsto l(a)$ are continuous. By the theorem of maximum, $q($.$) and l($.$) are continuous at any a=(x, y, \rho, \theta)$ with $\theta<1$. Now let $a_{n}=\left(x_{n}, y_{n}, \rho_{n}, \theta_{n}\right) \rightarrow \tilde{a}=(\tilde{x}, \tilde{y}, \tilde{\rho}, 1)$. Let $(\bar{q}, \bar{l})$ be an arbitrary limit point of $\left\{\left(q\left(a_{n}\right), l\left(a_{n}\right)\right)\right\}$. Because $(17)-(18)$ hold for $(q, l, a)=$ $\left(q\left(a_{n}\right), l\left(a_{n}\right), a_{n}\right)$, they hold for $(q, l, a)=(\bar{q}, \bar{l}, \tilde{a})$. Because $(q(\tilde{a}), l(\tilde{a}))$ is the unique $(q, l)$ satisfying (17)-(18) when $a=\tilde{a},(\bar{q}, \bar{l})=(q(\tilde{a}), l(\tilde{a}))$. Hence $q($. and $l($.$) are continuous, and so are f($.$) and g($.$) .$

Next, adding $(\theta, \tau)$ into the list of arguments of $v($.$) , we have mappings$ $(x, \rho, \mu, \theta, \tau) \mapsto v(x, \rho, \mu, \theta, \tau),(\rho, \mu, \theta, \tau) \mapsto X(\rho, \mu, \theta, \tau)$, and $(\rho, \mu, \theta, \tau) \mapsto$ $T(\rho, \mu, \theta, \tau)$ (for $T$, see (15) and (16)). By continuity of $f$ and $g$, I can show by the exact argument in the proof of Lemma 2 that $T$ is u.h.c. and compact-valued. Moreover, $(\rho, \mu, \theta, \tau) \mapsto W(\rho, \mu, \theta, \tau)$ is continuous, where $W(\rho, \mu, \theta, \tau)$ is the young person's expected lifetime utility in the steady state $(\rho, \mu)$ under $(\theta, \tau)$.

Next, by step-1 results, there exists $\tilde{\tau}>0$ such that $W(\tilde{\rho}, \tilde{\mu}, 1, \tilde{\tau})>$ $W(\rho, \mu, 1, \tau), \forall \tau \leq 0$. Suppose $\bar{\theta}$ does not exists. Then we can find $1>$ $\theta_{n} \rightarrow 1,0 \geq \tau_{n} \rightarrow \bar{\tau},\left(\rho_{n}, \mu_{n}\right)=s_{n}$, and $\left(\rho_{n}^{\prime}, \mu_{n}^{\prime}\right)=s_{n}^{\prime}$ such that $s_{n} \in$ $T\left(s_{n}, \theta_{n}, \tau_{n}\right), s_{n}^{\prime} \in T\left(s_{n}^{\prime}, \theta_{n}, \tilde{\tau}\right)$, and $W\left(s_{n}, \theta_{n}, \tau_{n}\right)>W\left(s_{n}^{\prime}, \theta_{n}, \tilde{\tau}\right)$. Referring to the proof of Proposition $1,\left\{\left(s_{n}, s_{n}^{\prime}\right)\right\}$ is in some compact $\boldsymbol{\rho} \times \boldsymbol{\mu}$, so it has a limit point $\left(s, s^{\prime}\right)$. Because $T$ is u.h.c. and compact-valued, $s \in T(s, 1, \bar{\tau})$ and $s^{\prime} \in T\left(s^{\prime}, 1, \tilde{\tau}\right)$. Because $W$ is continuous, $W(s, 1, \bar{\tau}) \geq W\left(s^{\prime}, 1, \tilde{\tau}\right)$. By step- 1 results, $s$ is the unique steady state under $(1, \bar{\tau})$. Also, $s^{\prime}$ is the unique steady state under $(1, \tilde{\tau})$, that is, $s^{\prime}=(\tilde{\rho}, \tilde{\mu})$. But because $\bar{\tau} \leq 0$, $W(s, 1, \bar{\tau}) \geq W\left(s^{\prime}, 1, \tilde{\tau}\right)$ leads to a contradiction.

\section{Comparison to the Lagos-Wright model}

I first sketch a version of the LW model. At stage 1 of date $t$, people meet in a centralized market. At stage 2 of date $t$, people meet in pairs in a decentralized market, and each person has an equal chance to be a buyer or a seller. The individual stage- $i$ utility from consuming $q$ is $\tilde{u}_{i}(q)$ and disutility from producing $q$ is $\tilde{c}_{i}(q)$. The discount factor between dates is $\beta \in(0,1)$ (there is no within-date discount). The special assumption is either $\tilde{u}_{1}(q)=q$ or $\tilde{c}_{1}(q)=q$; here I assume $\tilde{u}_{1}(q)=q$. The initial money stock $M_{0}$ is evenly distributed among people at the start of date 0 . In the date $t$ centralized market, each person receives $\tau M_{t}$ with $\tau>\beta-1$ units of money transfer. The centralized trade is competitive. The surplus from pairwise trade is split by generalized Nash bargaining in that the buyer's bargaining power is $\theta$. The equilibrium is a positive sequence of $\left\{\rho_{t}\right\}$ such that when $\rho_{t} M_{t}$ is the price 
of money in the date- $t$ centralized market, all people leave the market with $(1+\tau) M_{t}$ units of money.

For comparison, it is convenient to assume that in the OLG model, people can consume in their first stage with the utility function $u_{1}$ (which does not affect results in the main text). Let $\left(u_{i}, c_{i}\right)=\left(\tilde{u}_{i}, \tilde{c}_{i}\right), i=1,2$, and $u_{3}=\beta u_{1}$. Let the buyer's bargaining power be $\theta$. Let the rate of money transfer be $\tau$.

Let $\left\{\rho_{t}\right\}$ be an equilibrium in the LW model. It is straightforward to show that in the OLG model, $\left\{\left(\rho_{t}, \mu_{t}\right)\right\}$ with supp $\mu_{t}=\{1\}$ is an equilibrium. Evidently, the prices of money in the centralized market and the trade outcomes in the decentralized market in these two equilibria are identical. This is observational equivalence.

In the LW model, there exists a unique steady state under $\tau$ with $\rho_{t}=\tilde{\rho}(\tau)$ all $t$ (see [8]). It is straightforward to show

$$
V\left(\tau_{2}\right)-V\left(\tau_{1}\right)=(1-\beta)\left[\tilde{V}\left(\tau_{2}\right)-\tilde{V}\left(\tau_{1}\right)\right]+(1-\beta)\left[\tilde{\rho}\left(\tau_{1}\right)-\tilde{\rho}\left(\tau_{2}\right)\right],
$$

where $\tilde{V}(\tau)$ is the representative agent's steady-state expected discounted utility under $\tau$ in the LW model, and $V(\tau)$ is the young person's steadystate expected lifetime under $\tau$ in the OLG model. Now suppose in the LW model, $M_{0}$ is sold by the government to people in the date 0 centralized market (this does not affect the definition of equilibrium and observational equivalence); then $V\left(\tau_{2}\right)-V\left(\tau_{1}\right)=(1-\beta)\left[\tilde{V}\left(\tau_{2}\right)-\tilde{V}\left(\tau_{1}\right)\right]$, that is, $\tilde{V}$ and $V$ are maximized by the same rate of money transfer.

\section{References}

[1] A.B. Abel, Optimal monetary growth, J. of Monetary Economics 19 (1987), 437-450.

[2] C. Aliprantis, K. Border, "Infinite dimensional analysis, a hitchhiker's guide," Second Edition, Springer-Verlag, New York, 1999.

[3] B. Aruoba, R. Wright, Search, money, and capital, a neoclassical dichotomy, J. of Money, Credit and Banking 35 (2003), 1085-1105.

[4] J. Chiu and M. Molico, Idiosyncratic uncertainty, inflation, and welfare, mimeo, 2007. 
[5] A. Deviatov, Money creation in a random matching model, Topics in Macroeconomics 6 (2006), Issue 3, Article 5.

[6] A. Deviatov, N. Wallace, Another example in which money creation is beneficial, Advances in Macroeconomics 1 (2001), Issue 1, Article 1.

[7] D. Levine, Asset trading mechanisms and expansionary policy, J. of Econ. Theory 54 (1991), 148-164.

[8] R. Lagos, R. Wright, A unified framework for monetary theory and policy analysis, J. of Polit. Economy 113 (2005), 463-484.

[9] Y. Maeda, Fiat money in a pairwise-trading, multi-good, overlapping generations model, J. of Econ. Theory 54 (1991), 84-97.

[10] B. McCallum, The role of overlapping-generations models in monetary economics, Carnegie-Rochester Conference Series on Public Policy 18 (1983), 9-44.

[11] M. Molico, The distribution and prices in search equilibrium, Int. Econ. Review 47 (2006), 701-722.

[12] S. Russell, Random-matching money demand in an overlapping generations model, mimeo, 2005.

[13] J. Tobin, Money and economic growth, Econometrica 33 (1965), 671684 .

[14] J. Tobin, "The overlapping generations model of fiat money: discussion," Kareken and Wallace (eds.), Models of Monetary Economics, Minneapolis: Federal Reserve Bank of Minneapolis (1980), 83-90.

[15] S. Williamson and R. Wright, Barter and monetary exchange under private information, American Economic Review 84 (1994), 104-123.

[16] T. Zhu, N. Wallace, Pairwise trade and coexistence of money and higher return assets, J. of Econ. Theory 133 (2007), 524-535. 